\title{
BEPU ROBUSTNESS ANALYSIS VIA PERTURBED LAW-BASED SENSITIVITY INDICES
}

\author{
B. Iooss ${ }^{12}, \mathrm{~V}$. Vergès ${ }^{1}$ and V. Larget ${ }^{3}$ \\ ${ }^{1}$ EDF R\&D, 6 Quai Watier, 78401, Chatou, France \\ 2 Institut de Mathématiques de Toulouse, Université Paul Sabatier, \\ Toulouse, France \\ ${ }^{3}$ EDF DT, 19 rue Pierre Bourdeix, 69007 Lyon, France
}

bertrand.iooss@edf.fr, vanessa.verges@edf.fr, vincent.larget@edf.fr

\begin{abstract}
The "best-estimate plus uncertainty" (BEPU) methodology is the term used in the nuclear engineering community when dealing with uncertainty quantification issues in realistic numerical simulation models. One of the most critical hypothesis in these studies is the choice of the probability distributions of uncertain input variables which are propagated through the model. Bringing stringent justifications to the BEPU approach, especially in a safety study, requires quantifying the impact of potential uncertainty on the input variable distribution. To solve this problem, this paper deepens the robustness analysis based on the "Perturbed Law-based sensitivity Indices" (PLI). The PLI quantifies the impact of a perturbation of an input distribution on the quantity of interest (as a quantile of a model output or a safety margin) in the BEPU study. The mathematical formalism of the PLI is applied to two particular quantities of interest: the quantile and the superquantile. For both quantities, the PLI can be easily computed using a unique Monte-Carlo sample containing model inputs and output. Numerical tests are developed in order to define validity criteria of a PLI-based robustness analysis. The practical use of the method is illustrated on thermal-hydraulic computer experiments, simulating a cold leg Intermediate Break Loss Of Coolant Accident (IBLOCA) in a pressurized water nuclear reactor.
\end{abstract}

Keywords: Computer experiments, Density perturbation, Importance sampling, Nuclear safety, Sensitivity Analysis, Uncertainty

\section{Introduction}

Uncertainty quantification from numerical simulation models $[1,2,3]$ is gaining more and more attention in the engineering fields (e.g., nuclear and 
aerospace). It not only allows to assess the reliability and safety of complex systems (e.g., by estimating statistical risk measures as variance, quantile, probability of threshold exceedance, ...), but also to provide robust methodological tools which can complete the deterministic (and sometimes over-conservative) analyzes already performed, in order to better identify the safety margins. As an example, safety analyzes of nuclear power plants involving thermal-hydraulic issues, such as a cold leg Intermediate-Break Loss-Of-Coolant Accident (IBLOCA) in a pressurized water reactor, can be treated using probabilistic and statistical approaches $[4,5]$. In the nuclear engineering community, when the computer code, that is used to simulate the involved physical phenomena, lies on realistic assumptions, the uncertainty quantification framework is known as the "best-estimate plus uncertainty" (BEPU) methodology [6].

It is widely recognized that global sensitivity analysis plays a key role in the BEPU methodology $[7,8]$ and, more widely, in systems modeling and policy support $[9,10,11]$. When the quantity of interest is related to a rare event simulated by the numerical model, reliability-oriented sensitivity analysis methods can be put in action in order to quantify the sources of uncertainty (the model inputs) that drive the rare event [12, 13, 14, 15, 16]. However, one of the most critical hypothesis in classical BEPU studies is the choice of the probability distributions of the uncertain inputs which are propagated through the numerical model $[17,18]$.

When experimental data are available, the methodology called inverse uncertainty quantification allows to obtain the probabilistic distributions of some physical model inputs [17]. The inverse uncertainty quantification relies on a statistical inference process, involving a frequentist (via maximum likelihood inference) or a Bayesian approach [19, 20, 21]. Alternative approaches exist, as combining the use of design of experiments and forward uncertainty propagation (see [22,23] for examples in the nuclear field). For inputs without associated experimental data allowing to model their uncertainties, expert judgment is most often used. Whatever the method used for the choice of the probability density functions (pdf) for the model inputs, these pdf are established with various levels of confidence. In this paper, we focus on this particular issue associated to the residual uncertainty about each input pdf (whatever it was obtained). Indeed, facing to regulatory authorities, bringing stringent justifications to the BEPU approach requires quantifying the impact of this second-level uncertainty (i.e. the uncertainty on the input pdf) on the specific quantity of interest (QoI), most often a 
high-order quantile, related to the BEPU study.

To address such issues, several strategies exist in the literature as a probabilistic modeling of uncertainty in the parameters of the inputs' distributions (see [24] for the uncertainty propagation issues and [25, 26] for global sensitivity analysis tools in this context) or getting out of the probabilistic framework by dealing with imprecise probabilities theories (see [27] for a review and [28] for global sensitivity analysis in the context of the p-box theory). Recently, the new branch of robustness analysis in uncertainty quantification has emerged in the field of sensitivity analysis of model outputs [29, 30, 31]. It consists of evaluating the impact of the choice of the inputs' distributions and, more precisely, by analyzing the QoI variations with respect to this choice. Two practical interests of this approach are to stay within the probabilistic framework and to avoid the difficult step of probabilistically modeling the input distributions parameters. These two aspects are clearly advantageous for the industrial issues related to our IBLOCA application of interest [32].

One particularly interesting solution for the robustness analysis has been proposed in the context of reliability-oriented sensitivity analysis by [33] with the Perturbed Law-based sensitivity Indices (PLI). This measure only needs a Monte Carlo sample of model inputs and output, and presents the important advantage to be not limited by the number of model inputs that can be considered. Another advantage of the PLI is that it explicitly focuses on a particular QoI, that has to be specified by the user. The QoI of most BEPU studies is a high-order (e.g. 95\%) quantile of the model output variable $[4,34,32]$. However, in simulation-based risk analysis, several works [35, 36] have shown the interest of also considering the superquantile (i.e. the mean of the pdf tail exceeding the quantile) as a QoI. This risk measure gives interesting information about what is happening in the distribution-tail above the quantile. Its estimation also presents more regularities than those of the quantile estimation.

In this paper, the PLI-quantile concept [37, 38, 39] is extended to the PLIsuperquantile one. Via intensive simulation studies, our work also addresses a fundamental question that arises in practice: which level of perturbations can be applied on an input pdf so that the method remains valid? The following section develops the mathematical formalism of the PLI applied to the quantile and superquantile. It shows how, for both the QoI, the PLI can be easily computed using a unique Monte-Carlo sample (ensemble of code runs) containing model inputs and output. Section 3 describes the 
numerical tests that allow to define validity criteria of a PLI-based robustness analysis. Section 4 illustrates the PLI approach to assess the robustness of the IBLOCA thermal-hydraulic simulation scenario that uses the calculation code CATHARE2. A conclusion section ends this paper.

\section{Principles of PLI-quantiles and PLI-superquantiles}

\subsection{Introduction to PLI}

Given the random vector $\mathbf{X}=\left(X_{1}, \ldots, X_{d}\right) \in \mathcal{X} \subseteq \mathbb{R}^{d}$ of $d$ independent uncertain input variables of pdf $f(\mathbf{x})=f_{1}\left(x_{1}\right) \times \ldots \times f_{d}\left(x_{d}\right), G(\cdot)$ a numerical model and $Y=G(\mathbf{X}) \in \mathbb{R}$ the model output, the QoI is a statistical quantity derived from $Y$. For instance, it can be the mean, the variance, a quantile

or a threshold exceedance probability of the model output. In this paper, we limit the PLI analysis in the case of independent inputs and let to further studies the development of PLI in the dependent inputs case.

A PLI aims to measure the impact of the modification of an input density on a QoI [33]. In order to compute the $i$-th PLI, we change the density $f_{i}$ of $X_{i}$ into a density $f_{i \delta}$, where $\delta \in \mathbb{R}$ represents the level of the perturbation. The PLI is then simply defined as the relative change in the QoI generated by the perturbation:

$$
S_{i \delta}=\frac{\mathrm{QoI}_{i \delta}-\mathrm{QoI}}{\mathrm{QoI}}
$$

This definition slightly differs from the one proposed in previous studies $[33,38]$. Indeed, after several applications of the PLI, it has been found more convenient to compute directly the relative variation of the quantile when submitted to a density perturbation [32]. The interpretation of PLI is then straightforward.

\subsection{Quantiles and superquantiles}

The quantile of order $\alpha$ of $Y$ is

$$
q^{\alpha}=q^{\alpha}(Y)=\inf \left\{t \in \mathbb{R}, F_{Y}(t) \geq \alpha\right\}
$$

where $F_{Y}$ is the cumulative distribution function of the random variable $Y$. In BEPU studies, a high-order (e.g. 95\%) quantile value is most often preferred to a failure probability computation, as it can easily be related to safety margin concepts [4]. 
However, the quantile does not give any information about what is happening in the distribution-tail above the quantile. Another quantity, called superquantile, giving more information about the distribution-tail has therefore been introduced [40]. A superquantile of order $\alpha$ is defined by

$$
Q^{\alpha}=Q^{\alpha}(Y)=\mathbb{E}\left(\frac{G(\mathbf{X}) \mathbb{1}_{G(\mathbf{X}) \geq q^{\alpha}}}{1-\alpha}\right),
$$

where $\mathbb{E}(\cdot)$ denotes the expectation operator and $\mathbb{1}_{A}$ is the indicator function of the set $A$. If $F_{Y}$ is continuous, we have the equivalence with the following definition [41]:

$$
Q^{\alpha}=\mathbb{E}\left(G(\mathbf{X}) \mid G(\mathbf{X}) \geq q^{\alpha}\right) .
$$

Also known under the name of expected-shortfall and conditional-value-atrisk in finance, the superquantile has been proven to be a coherent risk measure (which is not the case for the quantile) [40,35]. Moreover, its empirical estimator often presents more regularities than those of the quantile empirical estimator.

\subsection{Reverse importance sampling-based estimation formulas}

In a lot of applications, for instance in nuclear safety studies (see, e.g., [42]), the computer models are costly in terms of CPU time and memory. Only a limited number $N$ of code runs is then available for the estimation of all the PLIs. The sample $\mathcal{X}_{N}=\left\{\mathbf{x}^{(n)}=\left(x_{1}^{(n)}, \ldots, x_{d}^{(n)}\right)\right\}_{1 \leq n \leq N}$ of $N$ independent realizations of $\mathbf{X}$ (which follow the pdf $f$ ) is propagated through the model $G$ to produce the sample $\mathcal{Y}_{N}=\left\{y_{n}\right\}_{1 \leq n \leq N}$ of $N$ model outputs. The most standard estimation of a quantile is based on the empirical quantile estimator denoted $\widehat{q}_{N}^{\alpha}=\inf \left\{t \in \mathbb{R}, \widehat{F}_{Y}^{N}(t) \geq \alpha\right\}$ where $\widehat{F}_{Y}^{N}(t)=\frac{1}{N} \sum_{n=1}^{N} \mathbb{1}_{y_{n} \leq t}$ is the empirical estimator of the cumulative density function of $Y$. The Wilks formula or the bootstrap technique can then be used to add a second level of conservatism (due to the limited size of the sample [43]). In this work, the bootstrap technique will be used.

From (2), the perturbed quantile writes:

$$
q_{i \delta}^{\alpha}=\inf \left\{t \in \mathbb{R}, F_{Y, i \delta}(t) \geq \alpha\right\},
$$

with $F_{Y, i \delta}$ the cumulative distribution function corresponding to the input variable $X_{i}$ sampled from $f_{i \delta}$. In order to give an estimation of the perturbed 
quantile (denoted $\widehat{q}_{N, i \delta}^{\alpha}$ ) from the same sample $\mathcal{X}_{N}$, we use the so-called reverse importance sampling mechanism [44] to compute $\widehat{F}_{Y, i \delta}^{N}$ [39]:

$$
\widehat{F}_{Y, i \delta}^{N}(t)=\frac{\sum_{n=1}^{N} L_{i}^{(n)} \mathbb{1}_{\left(y_{n} \leq t\right)}}{\sum_{n=1}^{N} L_{i}^{(n)}}
$$

with $L_{i}^{(n)}$ the likelihood ratio $\frac{f_{i \delta}\left(x_{i}^{(n)}\right)}{f_{i}\left(x_{i}^{(n)}\right)}$. We obtain

$$
\widehat{q}_{N, i \delta}^{\alpha}=\inf \left\{t \in \mathbb{R}, \widehat{F}_{Y, i \delta}^{N}(t) \geq \alpha\right\}
$$

The estimator $\widehat{S}_{N, i \delta}$ of the PLI-quantile consists in replacing QoI by $\widehat{q}_{N}^{\alpha}$ in Eq. (1). Its theoretical properties (asymptotic consistence and central limit theorem) have been given in [45].

From (3), the perturbed superquantile writes:

$$
Q_{i \delta}^{\alpha}=\mathbb{E}_{i \delta}\left(\frac{G(\mathbf{X}) \mathbb{1}_{G(\mathbf{X}) \geq q_{i \delta}^{\alpha}}}{1-\alpha}\right),
$$

where $\mathbb{E}_{i \delta}$ means that the expectation is taken under the perturbed distribution $\left(f_{1}, \ldots, f_{i \delta}, \ldots, f_{d}\right)$. We define an alternative version (used in the engineering study of [36]) of the perturbed superquantile:

$$
Q_{i \delta}^{\prime \alpha}=\mathbb{E}\left(\frac{G(\mathbf{X}) \mathbb{1}_{G(\mathbf{X}) \geq q_{i \delta}^{\alpha}}}{1-\alpha}\right) .
$$

This equation gives an alternative estimator because the expectation is taken under the initial distribution instead of the perturbed distribution. Its interest is that its estimation is easier and more stable (because the available sample is more representative of the initial distribution than the perturbed distribution). In the following, the alternative perturbed superquantile refers to Eq. (9), while the original perturbed superquantile refers to Eq. (8).

In order to estimate these quantities from the sample $\left(\mathcal{X}_{N}, \mathcal{Y}_{N}\right)$, we use the same mechanism (i.e. the reverse importance sampling procedure) as before by using the discretized-integral formula

$$
\widehat{Q}_{N, i \delta}^{\alpha}=\frac{1}{N(1-\alpha)} \sum_{n=1}^{N} G\left(\mathbf{x}^{(n)}\right) \mathbb{1}_{G\left(\mathbf{x}^{(n)}\right) \geq \widehat{q}_{N, i \delta}^{\alpha}} L_{i}^{(n)} .
$$


The estimator $\widehat{S}_{N, i \delta}$ of the original PLI-superquantile consists in replacing QoI (resp. $\operatorname{QoI}_{i \delta}$ ) by $\widehat{Q}_{N}^{\alpha}$ (resp. $\widehat{Q}_{N, i \delta}^{\alpha}$ ) in Eq. (1). For the alternative PLI-superquantile estimation, we simply write:

$$
{\widehat{Q^{\prime}}}_{N, i \delta}^{\alpha}=\frac{1}{N(1-\alpha)} \sum_{n=1}^{N} G\left(\mathbf{x}^{(n)}\right) \mathbb{1}_{G\left(\mathbf{x}^{(n)}\right) \geq \widehat{q}_{N, i \delta}^{\alpha}} .
$$

In the following sections, the PLI-quantile and original PLI-superquantile are considered. Moreover, the estimator $\widehat{S}_{N, i \delta}$ will be denoted $\widehat{S}_{i \delta}$.

\subsection{Input density perturbation method}

The density perturbation approach proposed in [33] (and used in [38, 46]) consists of replacing the density $f_{i}$ of one input $X_{i}$ by a perturbed one $f_{i \delta}$, where $\delta \in \mathbb{R}$ represents a shift of a moment (e.g. the mean or the variance). Amongst all densities with shifted mean or variance of a $\delta$ value, $f_{i \delta}$ is defined as the one minimizing the Kullback-Leibler divergence from $f_{i}$. Figure 1 illustrates some perturbed pdf after a perturbation on the mean of initial pdf. Increasing the mean of a Gaussian pdf just consists in translating it by a constant shift (the perturbed pdf remains Gaussian). Increasing the mean of a uniform pdf turns to a non-uniform pdf (because we force its support to be fixed) which gives more weights to the large values.

In the following, we focus on one type of perturbations used in most of the studies, that is the perturbation of the mean of the random variable. However, even in this simplest perturbation case, minimizing the KullbackLeibler divergence can be difficult or even impossible for some particular distributions (such as the log-normal one) [33]. A simpler approach proposed in [30] starts by applying an iso-probabilistic operator (such as the Rosenblatt transform [47]) to the initial input pdf, in order to transform all the input random variables into centered normalized Gaussian ones (denoted $\mathcal{N}(0,1)$ ). In case of independent inputs, it just corresponds to the inverse probability distribution transform. Then, the perturbations are applied on Gaussian pdf (as in Figure 1, left) and the solution is trivial as the perturbed pdf is $\mathcal{N}(\delta, 1)$ where $\delta$ is the perturbation level. This method allows to make perturbations comparable when applied in this standard space [39, 30, 32].

In practice, one has to define the variation range of $\delta$ as it can be of interest to decrease and increase it. Looking at the PLI evolution as a function of $\delta$ will be also informative. To illustrate this, we compute the PLI-quantile at order $\alpha=0.95$ on the linear model output $Y=2 X_{1}+X_{2}+X_{3} / 2$ with 

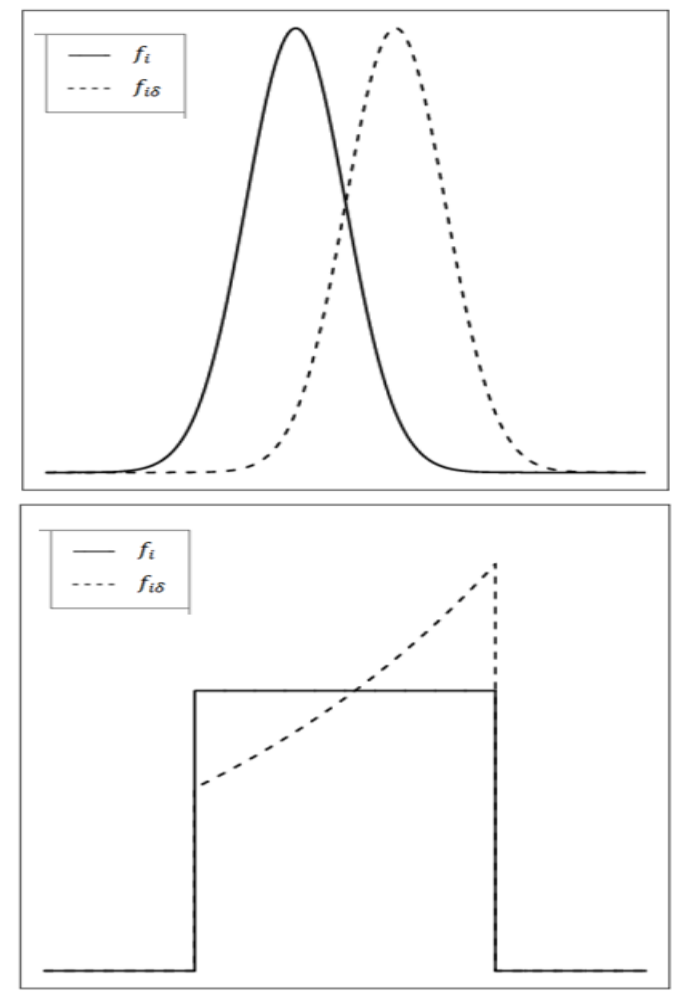

Figure 1: Examples of perturbed pdf for Gaussian (top) and uniform (bottom) initial pdf and a perturbation on the mean.

$X_{i} \sim \mathcal{N}(0,1)$ for $i=1,2,3$. The estimations are made by using a Monte Carlo sample $\left(\mathcal{X}_{N}, \mathcal{Y}_{N}\right)$ of size $N=5000$, by applying a mean perturbation on the range $\delta \in[-1,1]$ (with a regular discretization). Figure 2 shows the PLI curve for each input. In this linear model case, the PLI curves are linear because the output quantile increases linearly with the increase of each input mean. Moreover, the larger weight of $X_{1}$ in the model equation explains its larger impact than that of $X_{2}$ and $X_{3}$.

Thanks to the use of a Monte Carlo sample, applying a bootstrap technique (here with 200 replicas) allows to associate a confidence interval (CI), typically at $95 \%$, to each PLI estimate. Evaluating this estimation uncertainty is crucial in practice as the limited sample size can lead to erroneous result from a certain level of perturbation (e.g. when there are not enough sample values close to the perturbed quantile). For $\delta=0$, the PLI logically becomes null without any estimation error. 


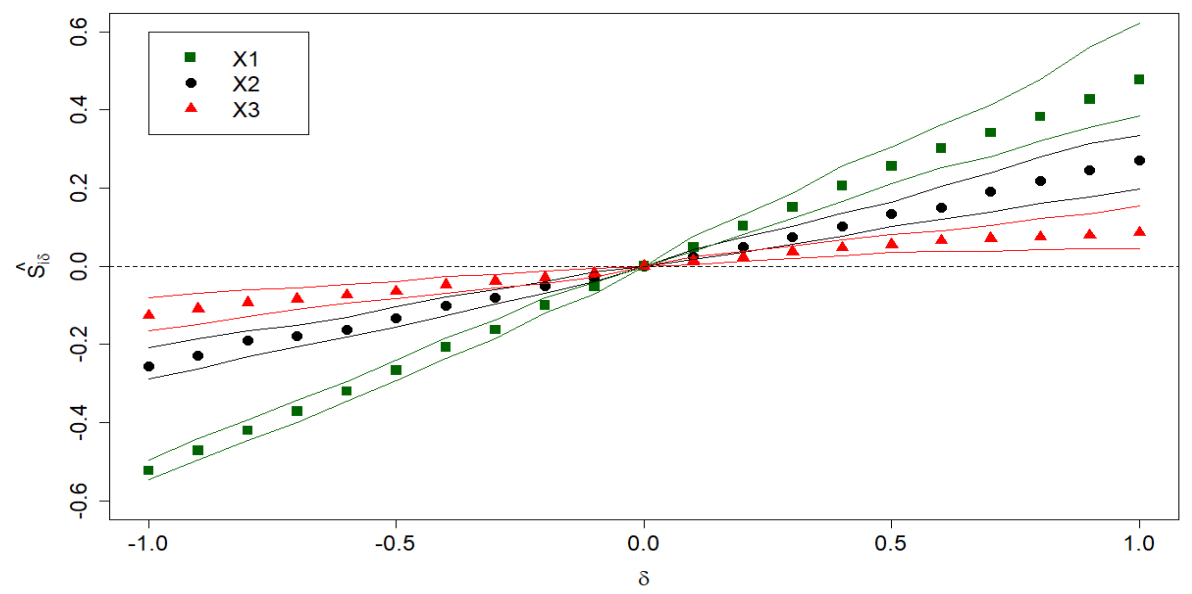

Figure 2: PLI-quantile $(\alpha=0.95)$ for the linear model. The points are the PLI at different discretized values of $\delta$. The lines are the corresponding bootstrap 95\%-confidence intervals.

\section{Numerical validation tests}

In order to validate the PLI-based robustness analysis, we develop in this section several numerical tests on toy functions with a small number of inputs (three inputs $X_{i} \sim \mathcal{N}(0,1)$ for $\left.i=1,2,3\right)$. Note that the method can be applied to large-dimensional models and non-Gaussian inputs as it will be shown in Section 4. The two toy models are the linear model shown in Section 2.4:

$$
Y=2 X_{1}+X_{2}+X_{3} / 2
$$

and the well-known (in global sensitivity analysis) Ishigami function:

$$
Y=\sin \left(X_{1}\right)+7 \sin ^{2}\left(X_{2}\right)+0.1 X_{3}^{4} \sin \left(X_{1}\right) \text {. }
$$

The PLI-quantile and (original) PLI-superquantile estimation (which are based on the same principles) are studied by applying a mean perturbation on the range $\delta \in[-3,3]$. In this section, the bootstrap-based 95\% PLI-CI are computed with $R=200$ replicas.

The Figure 3 shows the PLI-quantiles (first and second lines) and PLIsuperquantiles (third and fourth lines) with $\alpha=0.95$, for the input $X_{2}$ of the linear model 12, computed by using a Monte Carlo sample $\left(\mathcal{X}_{N}, \mathcal{Y}_{N}\right)$ of size $N=1000$ (first and third lines) and $N=10000$ (second and fourth lines). 
Results for $X_{1}$ and $X_{3}$ are not shown for sake of conciseness and as they are quite similar than those for $X_{2}$ (except than the PLI amplitudes are larger for $X_{1}$ and smaller for $X_{3}$ as preliminary shown in Fig. 2) due to their similar structural effects in the linear model (12).

The left column of Fig. 3 consists in comparing the PLI estimates in black (formulas of Section 2.3) with the reference results based on resimulations in red (i.e. results that are obtained without the reverse importance sampling procedure but by resampling the input following the perturbed pdf). Of course, in a real-case study, only the reverse sampling approach is applicable. Note that the CI of the PLI estimates are obtained by bootstrap (as in Fig. 2) while the CI of the reference results are obtained by 100 Monte Carlo repetitions of the resimulation process (so these CI are no subject to bootstrap-based approximation). We can observe that the PLI (and the PLI$\mathrm{CI}$ ) are valid in the central zone of the plots (centered on the value $\delta=0$ ) and turn to be invalid at large $\delta$ values (when red CI are not inside black CI). Moreover, increasing the sample size extends the PLI-CI validity domain with respect to the $\delta$ variation range. Indeed, as the sample size is finite, at a certain level of perturbation, there is not enough sample values to correctly compute the perturbed quantile and its CI.

From these findings, two heuristics are proposed to provide warnings to users when $\delta$ is too large:

1. The number of values in $\mathcal{X}_{N}$, smaller or larger of the $\delta$-perturbed mean of an input variable, has to be sufficient. A value of $N_{x}=30$ has been chosen (from several numerical tests) as the smallest size. This criterion is easily computed from $\mathcal{X}_{N}$ and each $\delta$-value. It is represented by the green vertical lines on each plot. Of course, this value of 30 is quiet subjective and will be subject to further sensitivity studies.

2. The number of values in $\mathcal{Y}_{N}$, smaller or larger of the $\delta$-perturbed quantile of the model output, has to be sufficient. A value of $N_{y}=5$ (resp. $N_{y}=10$ ) has been chosen (from several numerical tests) as the smallest size for the PLI-quantile (resp. PLI-superquantile). It is represented by the blue vertical line on each plot. Of course, these values of 5 and 10 are quiet subjective and will be subject to further sensitivity studies.

In Figure 3 (left column), these two criteria are visualized in each plot. It shows their relevance to warn the user that the PLI values (and their CI) no longer make sense beyond these limits (e.g. because PLI-CI do not contain 

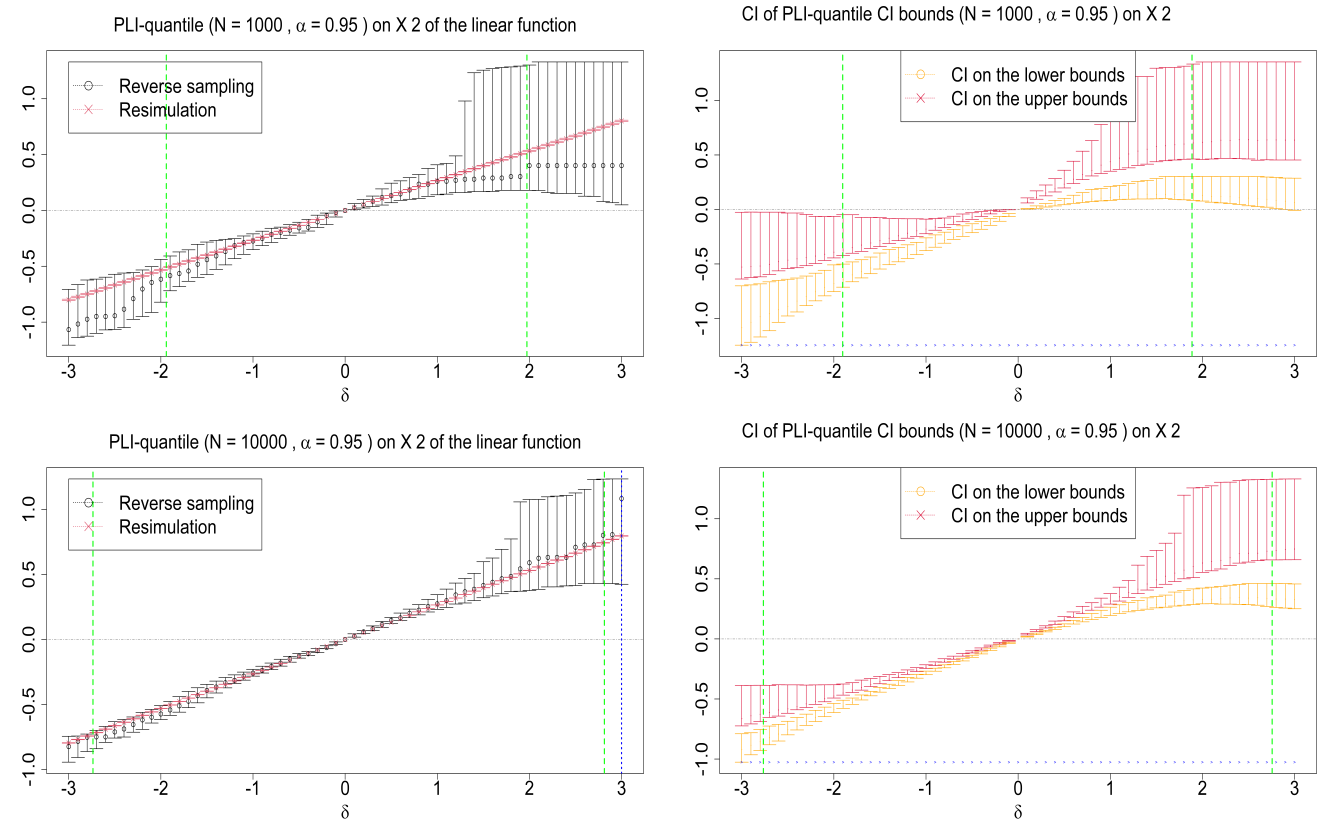

Cl of PLI-quantile $\mathrm{Cl}$ bounds ( $\mathrm{N}=10000, \alpha=0.95)$ on $\mathrm{X} 2$

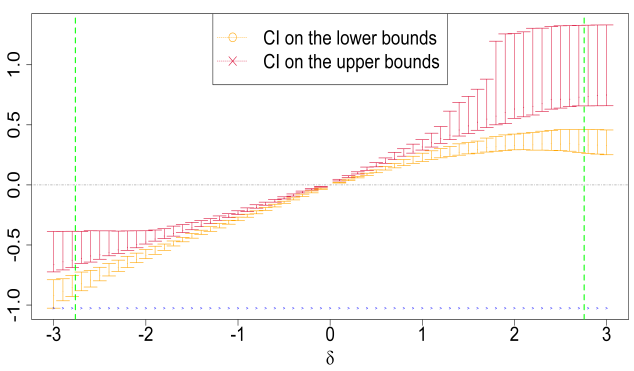

Cl of PLI-superquantile $\mathrm{Cl}$ bounds $(\mathrm{N}=1000, \alpha=0.95)$ on $\mathrm{X} 2$
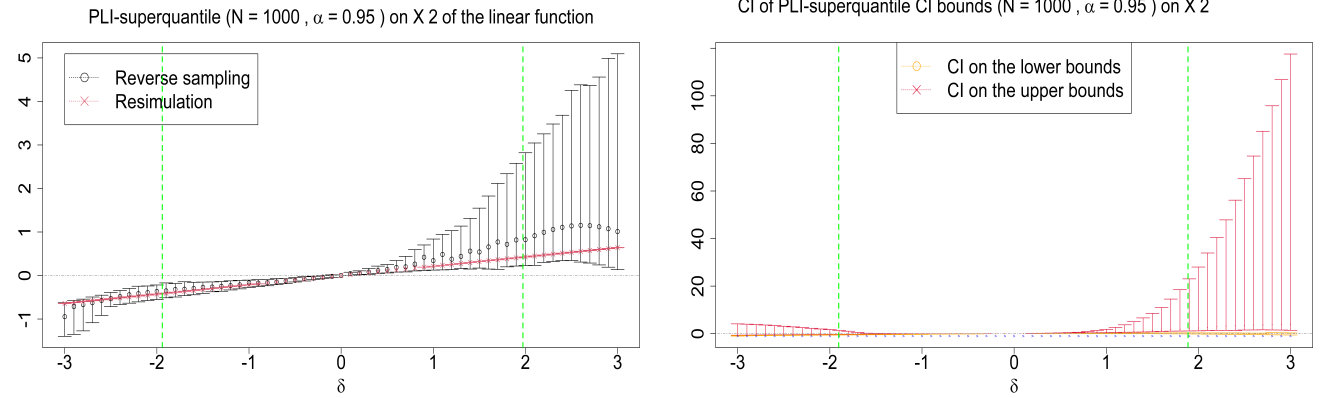

PLI-superquantile ( $N=10000, \alpha=0.95)$ on $\mathrm{X} 2$ of the linear function

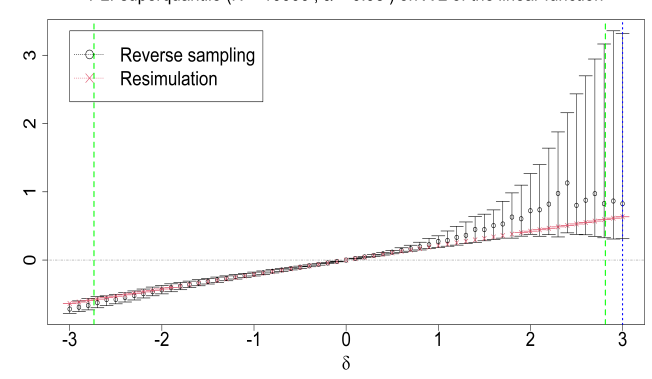

$\mathrm{Cl}$ of PLI-superquantile $\mathrm{Cl}$ bounds $(\mathrm{N}=10000, \alpha=0.95)$ on $\mathrm{X} 2$

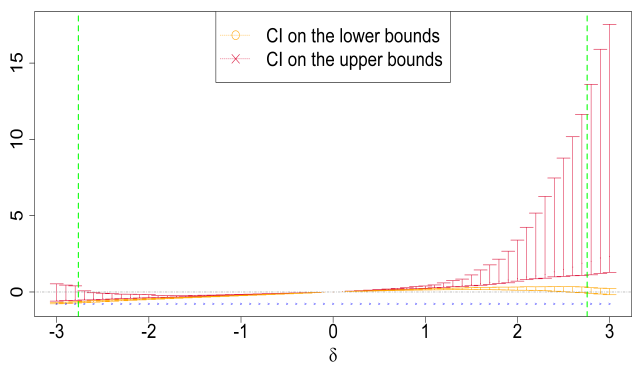

Figure 3: PLI validation results applied on $X_{2}$ of the linear model. 
the PLI reference values). Of course, the green and blue vertical lines are not visible in the plots when they are outside of the $\delta$ variation range.

The right column of Fig. 3 visualizes the uncertainty on the PLI-CI by showing the 95\%-CI of the PLI-CI lower bound (in orange) and the 95\%-CI of the PLI-CI upper bound (in red). These 95\%-CI are obtained by 50 Monte Carlo repetitions of the bootstrap-based estimation of the PLI-CI. It aims to visualize the stability of the PLI-CI, knowing that looking at the value of the PLI should not be done without looking at its associated CI. We verify that, beyond the limits given by the green and blue vertical lines, the CIs of the lower and upper bounds of the PLI-CI explode.

For the Ishigami function (13), the Figure 4 provides the results of the validity tests, computed by using a Monte Carlo sample $\left(\mathcal{X}_{N}, \mathcal{Y}_{N}\right)$ of size $N=1000$ and $N=10000$. The PLI-quantiles (first and second lines) and PLI-superquantiles (third and fourth lines), with $\alpha=0.95$, are given for $X_{1}$ (left column) and $X_{2}$ (right column). The interest of this toy case is to show that the PLI can also capture some strong non-linear behavior. Inside the bounds given by the green vertical lines (criteria on the sufficient number of points smaller or larger of the $\delta$-perturbed mean of the input variable), we observe the validity of the PLI-CI (the PLI-CI encompasses the red curve corresponding to resimulation which gives reference PLI values, i.e. estimates without bias). The blue vertical line does not appear on these plots, meaning that the number of output variable points smaller or larger of the $\delta$-perturbed quantile or superquantile is sufficient. We conclude that these results show

similar behavior than the linear model ones and confirm the relevance of the two heuristic validity criteria.

\section{Application on IBLOCA industrial use-case}

As a nuclear facility operator, EDF (the French company of electricity) has to perform safety analysis on his pressurized water nuclear reactors. Those studies include the "Loss of Coolant Accident" resulting in a cold leg intermediatebreak on the primary loop of the reactor (noted IBLOCA). This hypothetical scenario is simulated using system thermal-hydraulic computer codes, which include tens of physical parameters such as condensation or heat transfer coefficients [48, 49]. Yet, the values of the model parameters are known with a limited precision as they are evaluated through tests performed on smallscale experimental facilities. Some other variables are only observed during periodic inspections, such as the characteristics of hydraulics systems. 
PLI-quantile $(\mathrm{N}=1000, \alpha=0.95)$ on $\mathrm{X} 1$ of the Ishigami function

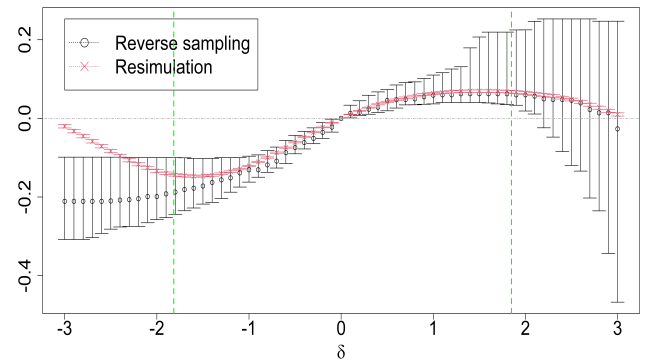

PLI-quantile ( $N=10000, \alpha=0.95)$ on $\mathrm{X} 1$ of the Ishigami function

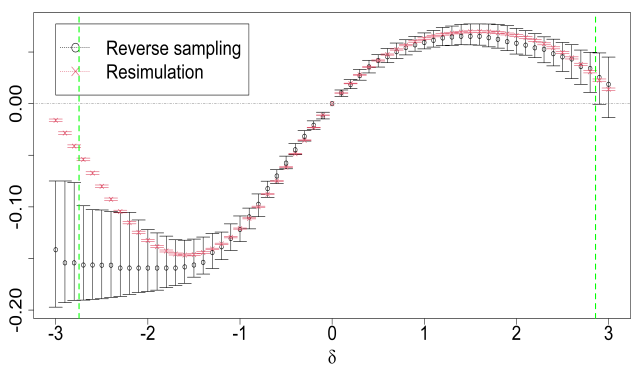

PLI-superquantile ( $N=1000, \alpha=0.95)$ on $\mathrm{X} 1$ of the Ishigami function

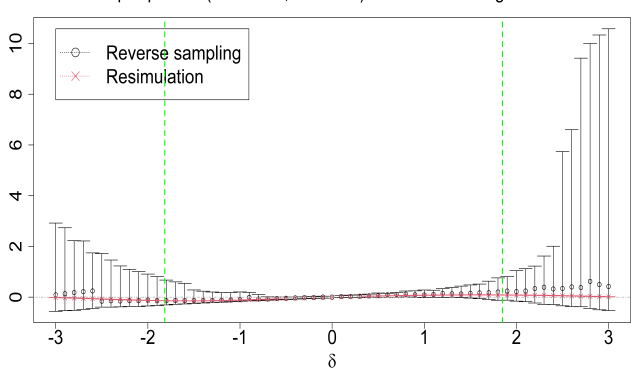

PLI-superquantile $(N=10000, \alpha=0.95)$ on $X 1$ of the Ishigami function

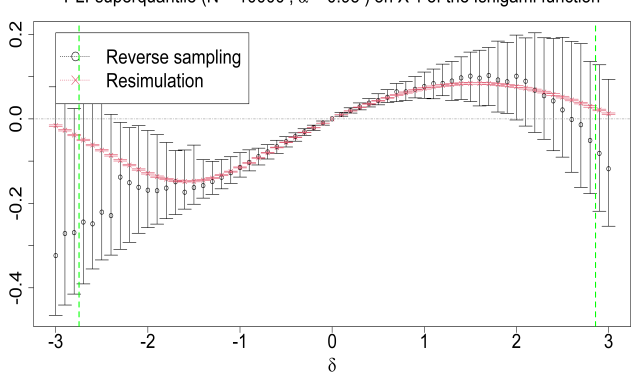

PLI-quantile $(\mathrm{N}=1000, \alpha=0.95)$ on $\mathrm{X} 2$ of the Ishigami function

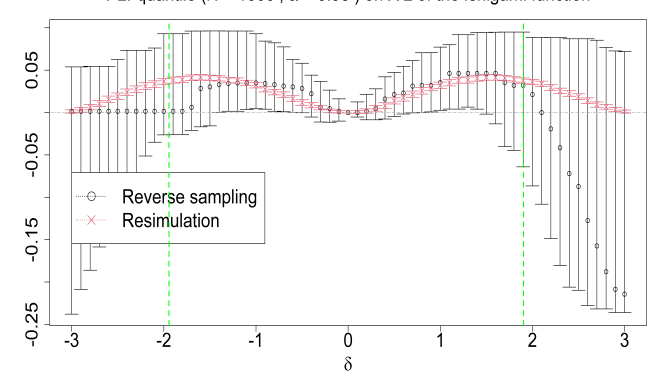

PLI-quantile $(\mathrm{N}=10000, \alpha=0.95)$ on $\mathrm{X} 2$ of the Ishigami function

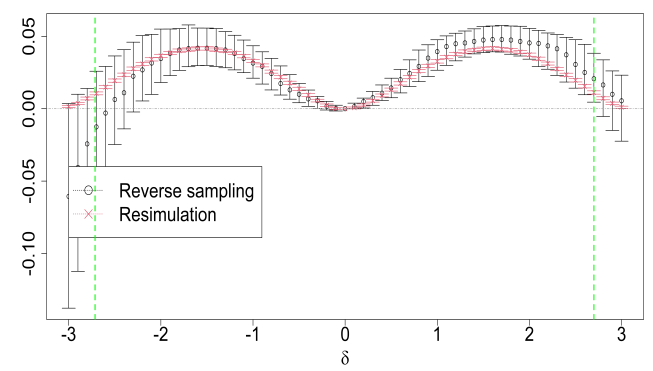

PLI-superquantile $(\mathrm{N}=1000, \alpha=0.95)$ on $\mathrm{X} 2$ of the Ishigami function

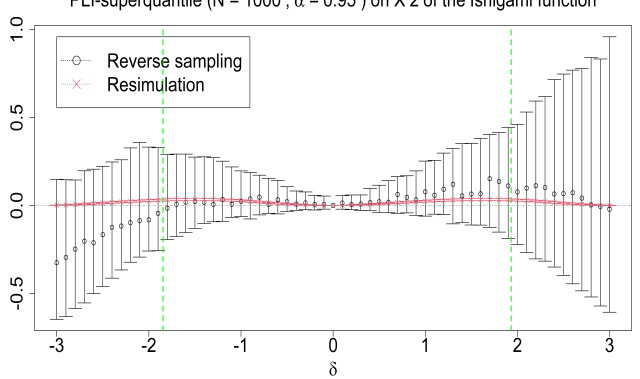

PLI-superquantile $(\mathrm{N}=10000, \alpha=0.95)$ on $\mathrm{X} 2$ of the Ishigami function

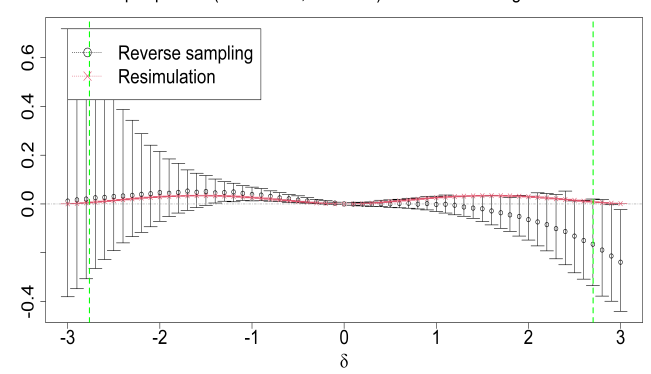

Figure 4: PLI validation results applied on $X_{1}$ and $X_{2}$ of the Ishigami function. 
The use-case of the present work is a 4-loop reactor from the French fleet ${ }^{1}$. The transient studied is an IBLOCA, the break considered is a 13-inches break located on the cold leg. The reactor is supposed to be at nominal power at the opening of the break. The main common assumptions for IBLOCA safety analysis are considered. The availability of the systems is assumed to be restricted to the minimum safety systems (ECCS, EFWS, ... ), other systems are considered to be unavailable. The most limiting additional single failure is postulated.

The IBLOCA transients are computed using the CATHARE2 code (developed by CEA, EDF, Framatome and IRSN [50]), which simulates the time evolution of physical quantities during a thermal hydraulic transient. In our simulation scenario, $d \approx 100$ random inputs are considered and the output variable of interest is the second peak cladding temperature. For each input, depending on its nature and the information available on it, a pdf type (e.g. uniform, truncated Gaussian, truncated lognormal, triangular) is chosen, in concordance with its parameters. A Monte Carlo sample of $N=2000$ input and output values have then be generated.

The QoI for this study is 95\%-quantile and 75\%-superquantile of the peak cladding temperature, which is estimated from the Monte Carlo sample at $q^{0.95}=737^{\circ} \mathrm{C}$ and $Q^{0.75}=673^{\circ} \mathrm{C}$. Our PLI approach consists in transforming each input pdf in a $\mathcal{N}(0,1)$ one, then perturbing the mean of each input with $\delta \in[-1.64,1.64]$. This range corresponds to the $5 \%$-quantile and $95 \%$ quantile of a $\mathcal{N}(0,1)$ variable. Figure 5 illustrates several perturbed empirical pdf from the Monte Carlo sample for four different variables.

On this high dimensional industrial application case (100 inputs), all the PLI can be easily computed. For a sake of conciseness, only 8 inputs with strong influence (described in Table 1) are shown in Fig. 6 through their PLI-quantile and (original) PLI-superquantile curves. The bootstrap-based 95\% CI are computed with $R=50$ replicas. Validity criteria do not appear on the plots because the size of the Monte Carlo sample is sufficient with respect to the perturbation values that have been considered.

By comparing the results of PLI-quantile and PLI-superquantile, we observe that both PLI give the same trends. However, differences can be noted on the most influential parameters, depending on the level of perturbation $\delta$ reached. Furthermore, PLI clearly determine the penalizing variation di-

\footnotetext{
${ }^{1}$ The input data considered for this case, and thus the obtained peak cladding temperature, do not correspond to actual industrial values
} 


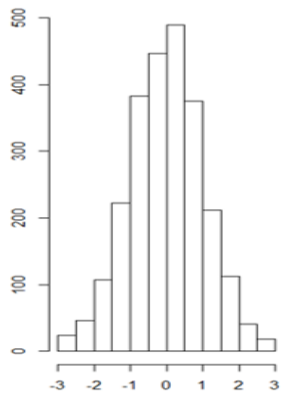

(a) Truncated Gaussian
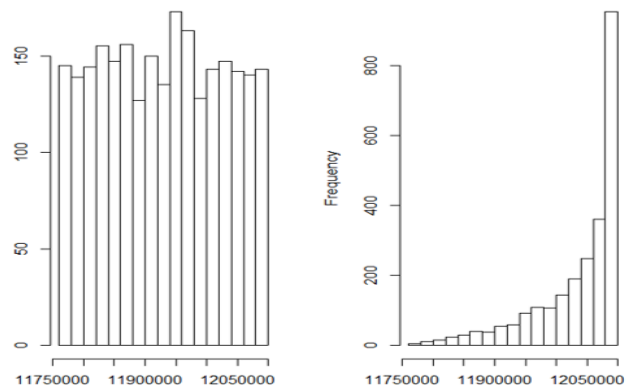

(c) Uniform
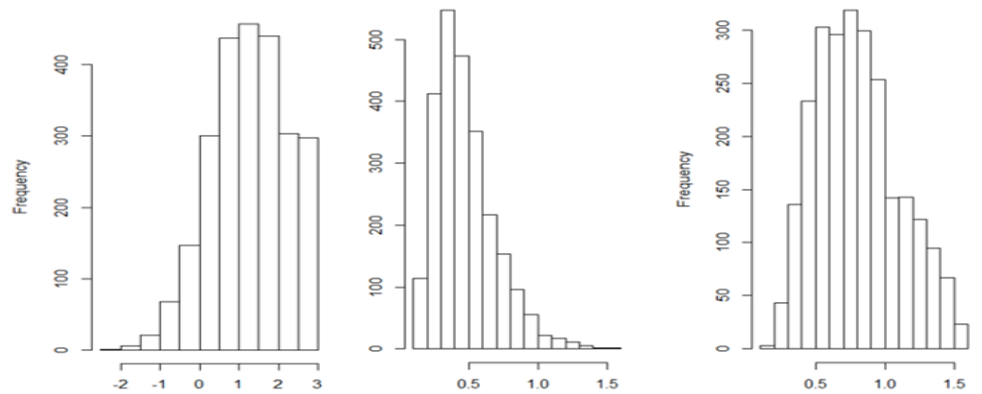

(b) Truncated lognormal
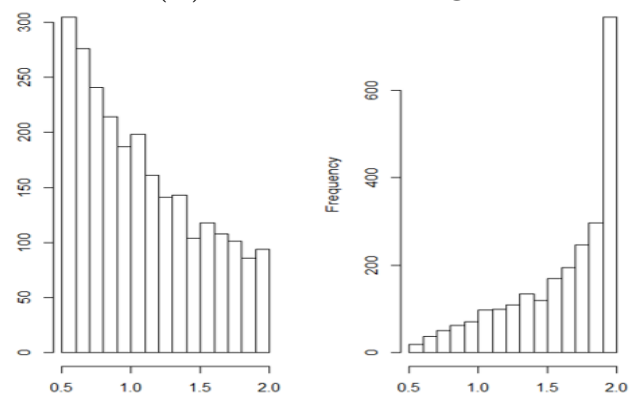

(b) Triangular

Figure 5: Examples of perturbed pdf for four inputs with different pdf of the IBLOCA case. Each case (a, b, c, d) shows the initial pdf (left) and the perturbed pdf by taking $\delta=1.28$ (right).

rection of each input: the QoI (0.95-quantile and 0.75-superquantile of the PCT) increase with an increase of the friction coefficients (X78, X110 and X111) and a decrease of the stable film temperature (X52), the exchange coefficients (X64 and X76) and the bubble-rise velocity X113. Decreasing X111 also leads to an increase of the QoI which allows to emphasize the non-monotonic behavior of the peak cladding temperature with respect to the interfacial friction in the core during blowdown phase. Such information is precious for discussing BEPU results.

In Figure 6, one important observation is the behavior of the confidence interval when $\delta$ is high. For some variables, for example X111 on the PLIquantile calculation, a saturation of the CI occur for high values of $\delta$. This is due to the limited number of value of the output exceeding the quantile, which is particularly large for X111. That behavior is not observed in the PLI-superquantile calculation because of the inherent regularity of the su- 


\begin{tabular}{|l|l|c|}
\hline Input & Variable description & Distribution \\
\hline X52 & Minimum Stable Film Temperature & Uniform \\
X64 & HTC wall-steam exchange in C7 zone & Log-Normal \\
X76 & HTC primary-secondary - Natural liquid & \\
& convection in SG & Log-Uniform \\
X78 & Wall-steam friction coefficient (CV) in HA & Log-Uniform \\
X110 & Interfacial friction in the downcomer & Log-Normal \\
X111 & Interfacial friction in the core during & \\
& blowdown phase & Log-Normal \\
X113 & Bubble-rise velocity in the upper plenum & Log-Normal \\
X116 & Bubble-rise velocity in the upper head & Log-Normal \\
\hline
\end{tabular}

Table 1: Definition of the 8 input variables considered for the PLI calculations. All the log-normal distributions are truncated.

perquantile, and the fact that the level of superquantile considered is low enough. This phenomenon seems to be fostered by the combination of PLIquantile at high quantile level and strong perturbation. Thus, for further industrial applications, in order to have the most accurate estimation of the PLI, the use of PLI-superquantile associated to limited value of $\delta$ (typically $\delta \leq 1$ ) should be considered, in addition to the application of the heuristic validity criteria. This recommendation has already been considered in the industrial methodology presented in [36].

\section{Conclusion}

In the context of uncertainty quantification studies and in particular when applying BEPU methodologies, hypotheses have to be made in order to define the input distributions which are not always easy to justify. This work has focused on the PLI approach which allows to challenge the hypotheses made on the uncertain input pdf. The main goal of PLI is to quantify the robustness of a BEPU QoI with respect to uncertainty on the pdf of the model inputs. However, PLI is also a sensitivity measure quantifying the influence of each input on the QoI. In this paper, PLI has allowed to quantify the impact of each input mean on quantiles and superquantiles. Finally, an important contribution of this work relies in the introduction of new criteria for studying the validity of the PLI analysis. 

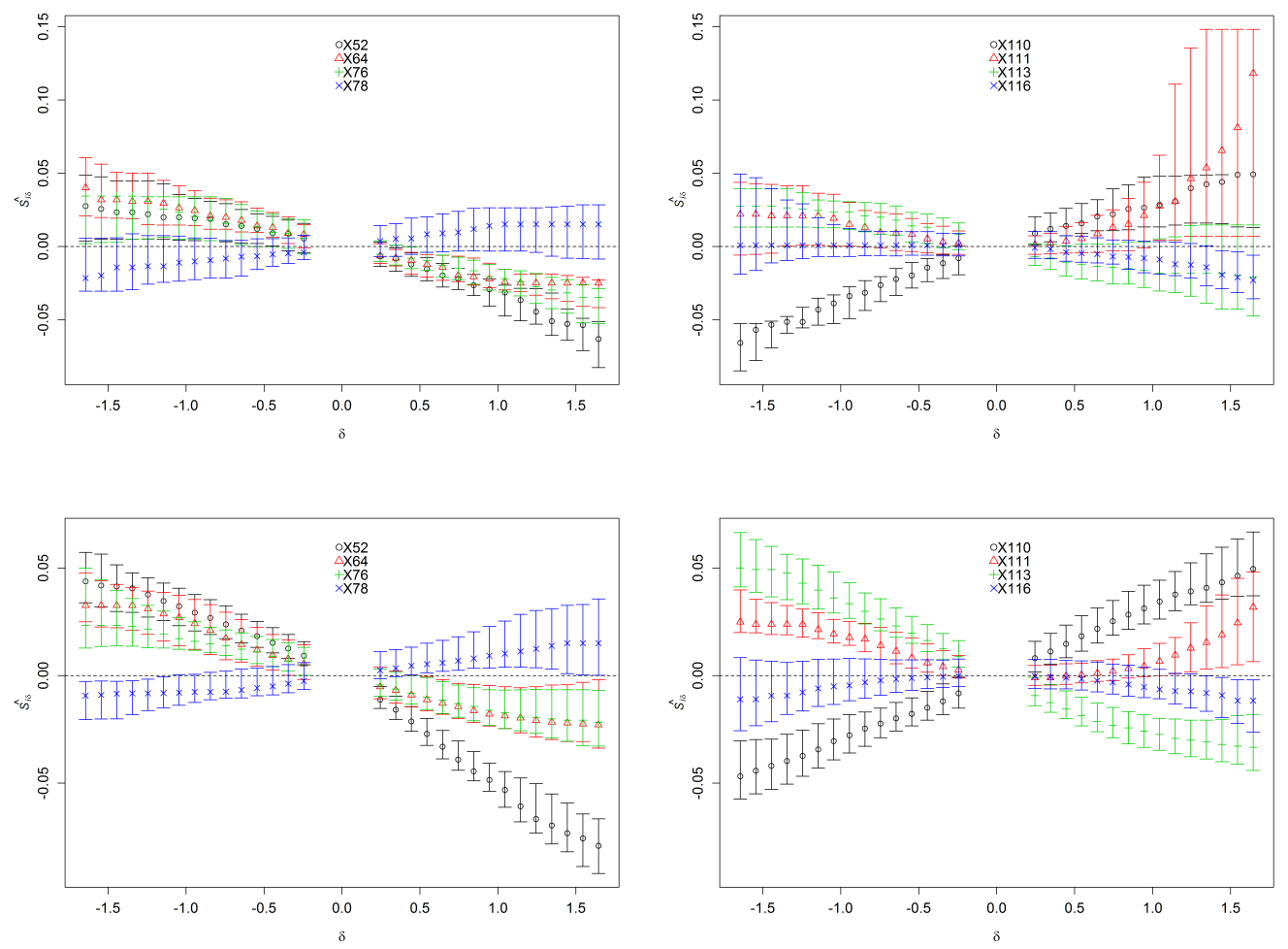

Figure 6: PLI-0.95-quantile (upper panels) and PLI-0.75-superquantile (lower panels) of the IBLOCA case.

This approach has been applied to an IBLOCA scenario (nuclear reactor thermal-hydraulic phenomena) simulated with the CATHARE2 code. In addition to the rich information that is provided on the inputs' influence and on the QoI robustness, it has enlightened the two important advantages of the PLI approach: it only needs a simple Monte Carlo sample and it is not limited in terms of number of model inputs. For further industrial applications, in order to have the most accurate estimation of the PLI, the use of PLI-superquantile associated to limited value of $\delta$ (typically less or equal to 1) should be considered.

To develop more deeply the PLI, further works have to focus on more general perturbation type (see, e.g., a first proposition in [45]), on developing multivariate PLI (perturbing several inputs at the same time), on considering the dependent inputs case (as in [51] in another methodological framework) 
and on using more efficient sampling technique than Monte Carlo scheme (as the importance sampling as preliminary studied in [37] for PLI estimation).

\section{Acknowledgments}

We are grateful to Jérôme Stenger for his help on theoretical aspects of PLIsuperquantiles. We also thank Thibault Delage and Roman Sueur for useful remarks, as well as two anonymous reviewers. Numerical computations of the PLI have been realized using the sensitivity package [52] of the R software.

\section{References}

[1] de Rocquigny E, Devictor N and Tarantola S (eds.) Uncertainty in industrial practice. Wiley, 2008.

[2] Smith R. Uncertainty quantification. SIAM, 2014.

[3] Morio J and Balesdent M. Estimation of rare event probabilities in complex aerospace and other systems. Woodhead Publishing, 2016.

[4] Prosek A and Mavko B. The state-of-the-art theory and applications of best-estimate plus uncertainty methods. Nuclear Technology 2007; 158: 69-79.

[5] Scarrott C, MacDonald A, Coolen F et al. Extreme-value-model-based risk assessment for nuclear reactors. Proceedings of the Institution of Mechanical Engineers, Part O: Journal of Risk and Reliability 2010; 224: 239-252.

[6] Wilson G. Historical insights in the development of Best estimate Plus Uncertainty safety analysis. Annals of Nuclear Energy 2013; 52: 2-9.

[7] de Crécy A, Bazin P, Glaeser H et al. Uncertainty and sensitivity analysis of the LOFT L2-5 test: Results of the BEMUSE programme. Nuclear Engineering and Design 2008; 12: 3561-3578.

[8] Baccou J, Zhang J, Fillion P et al. SAPIUM: A generic framework for a practical and transparent quantification of thermal-hydraulic code model input uncertainty. Nuclear Science and Engineering 2020; 194: 721-736. 
[9] Saltelli A, Ratto M, Andres T et al. Global sensitivity analysis - The primer. Wiley, 2008.

[10] Borgonovo E. Sensitivity Analysis - An Introduction for the Management Scientist. Springer, 2017.

[11] Razavi S, Jakeman A, Saltelli A et al. The future of sensitivity analysis: An essential discipline for systems modelling and policy making. Environmental Modelling and Software 2020; 137(104954).

[12] Zio E and Pedroni N. Monte Carlo simulation-based sensitivity analysis of the model of a thermal-hydraulic passive system. Reliability Engineering 8 System Safety 2012; 107: 90-106.

[13] Wei P, Song J and Lu Z. Global reliability sensitivity analysis of motion mechanisms. Proceedings of the Institution of Mechanical Engineers, Part O: Journal of Risk and Reliability 2016; 230: 265-277.

[14] Wei P, Tang C and Yang Y. Structural reliability and reliability sensitivity analysis of extremely rare failure events by combining sampling and surrogate model methods. Proceedings of the Institution of Mechanical Engineers, Part O: Journal of Risk and Reliability 2019; 233: 943-957.

[15] Marrel A and Chabridon V. Statistical developments for target and conditional sensitivity analysis: application on safety studies for nuclear reactor. Reliability Engineering \& System Safety 2021; 214: 107711.

[16] Zhang X, Lu Z, Cheng K et al. A novel reliability sensitivity method based on directional sampling and Monte Carlo simulation. Proceedings of the Institution of Mechanical Engineers, Part O: Journal of Risk and Reliability 2020; 234: 622-635.

[17] Baccou J, Zhang J, Fillion P et al. Development of good practice guidance for quantification of thermal-hydraulic code model input uncertainty. Nuclear Engineering and Design 2019; 354: 110173.

[18] Chabridon V, Balesdent M, Perrin G et al. "Global Reliability-oriented Sensitivity Analysis under Distribution Parameter Uncertainty". In Gogu C (ed.) Mechanical Engineering Under Uncertainties: From Classical Approaches to Some Recent Developments. Wiley - ISTE Ltd, pp. $237-277$. 
[19] Tarantola A. Inverse problem theory and methods for model parameter estimation. Society for Industrial and Applied Mathematics, SIAM, 2005 .

[20] Mendizábal R. Bayesian perspective in bepu licensing analysis. In Proceedings of ANS Best Estimate Plus Uncertainty International Conference (BEPU 2018). Lucca, Italy.

[21] Damblin G and Gaillard P. Bayesian inference and non-linear extensions of the CIRCE method for quantifying the uncertainty of closure relationships integrated into thermal-hydraulic system codes. Nuclear Engineering and Design 2020; 359: 110391.

[22] Kovtonyuk A, Lutsanych S, Moretti F et al. Development and assessment of a method for evaluating uncertainty of input parameters. In NURETH-16. Chicago, USA.

[23] Freixa J, de Alfonso E and Reventós F. Testing methodologies for quantifying physical models uncertainties. A comparative exercise using CIRCE and IPREM (FFTBM). Nuclear Engineering and Design 2016; 305: 653-665.

[24] Pasanisi A, Keller M and Parent E. Estimation of a quantity of interest in uncertainty analysis: Some help from Bayesian decision theory. Reliability Engineering 83 System Safety 2012; 100: 93-101.

[25] Chabridon V, Balesdent M, Bourinet JM et al. Reliability-based sensitivity estimators of rare event probability in the presence of distribution parameter uncertainty. Reliability Engineering 83 System Safety 2018; 178: $164-178$.

[26] Meynaoui A, Marrel A and Laurent B. New statistical methodology for second level global sensitivity analysis. Preprint 2019, https://hal.archives-ouvertes.fr/hal-02019412; .

[27] Beer M, Ferson S and Kreinovich V. Imprecise probabilities in engineering analyses. Mechanical Systems and Signal Processing 2013; 37: $4-29$. 
[28] Song J, Lu Z, Wei P et al. Global sensitivity analysis for model with random inputs characterized by probability-box. Proceedings of the Institution of Mechanical Engineers, Part O: Journal of Risk and Reliability 2015; 229: 237-253.

[29] Iooss B. Sensitivity analysis of model outputs: methods and issues for BEPU methodology. In Proceedings of ANS Best Estimate Plus Uncertainty International Conference (BEPU 2018). Lucca, Italy.

[30] Perrin G and Defaux G. Efficient estimation of reliability-oriented sensitivity indices. Journal of Scientific Computing 2019; 79: 1433-1455.

[31] Hart J and Gremaud P. Robustness of the Sobol' indices to marginal distribution uncertainty. SIAM/ASA Journal on Uncertainty Quantification 2019; 7: 1224-1244.

[32] Larget $\mathrm{V}$. How to bring conservatism to a BEPU analysis. In NURETH18. Portland, USA.

[33] Lemaître P, Sergienko E, Arnaud A et al. Density modification based reliability sensitivity analysis. Journal of Statistical Computation and Simulation 2015; 85: 1200-1223.

[34] Mousseau V and Williams B. Uncertainty quantification in a regulatory environment. In Ghanem R, Higdon D and Owhadi H (eds.) Springer Handbook on Uncertainty Quantification. Springer, pp. 1613-1648.

[35] Labopin-Richard T, Gamboa F, Garivier A et al. Bregman superquantiles. Estimation methods and applications. Dependence Modeling 2016; : 76-108.

[36] Larget V and Gautier M. Increasing conservatism in BEPU IB LOCA safety studies using complementary and industrially cost effective statistical tools. In e-proceedings of BEPU 2020 (cancelled conference).

[37] Sueur R, Bousquet N, Iooss B et al. Perturbed-law based sensitivity indices for sensitivity analysis in structural reliability. In Proceedings of the 8th International Conference on Sensitivity Analysis of Model Output (SAMO 2016). Le Tampon, Réunion Island, France. 
[38] Sueur R, Iooss B and Delage T. Sensitivity analysis using perturbedlaw based indices for quantiles and application to an industrial case. In Proceedings of the 10th International Conference on Mathematical Methods in Reliability (MMR 2017). Grenoble, France.

[39] Delage T, Sueur R and Iooss B. Robustness analysis of epistemic uncertainties propagation studies in LOCA assessment thermal-hydraulic model. In Proceedings of ANS Best Estimate Plus Uncertainty International Conference (BEPU 2018). Lucca, Italy.

[40] Rockafellar R. Coherent approaches to risk in optimization under uncertainty. Tutorials in Operations Research INFORMS 2007 2007; : $38-61$.

[41] Rockafellar R and Uryasev S. Conditional value-at-risk for general loss distributions. Journal of Banking \& Finance 2002; 26: 1443-1471.

[42] Iooss B and Marrel A. Advanced methodology for uncertainty propagation in computer experiments with large number of inputs. Nuclear Technology 2019; 205: 1588-1606.

[43] Zio E and Di Maio F. Bootstrap and order statistics for quantifying thermal-hydraulic code uncertainties in the estimation of safety margins. Science and Technology of Nuclear Installations 2008; 2008(Article ID 340164, 9 pages, DOI:10.1155/2008/340164).

[44] Hesterberg T. Estimates and confidence intervals for importance sampling sensitivity analysis. Mathl Comput Modelling 1996; 23: 79-85.

[45] Gauchy C, Stenger J, Sueur R et al. An information geometry approach for robustness analysis in uncertainty quantification of computer codes. Technometrics, In press 2021; DOI:10.1080/00401706.2021.1905072.

[46] Iooss B and Le Gratiet L. Uncertainty and sensitivity analysis of functional risk curves based on Gaussian processes. Reliability Engineering 63 System Safety 2019; 187: 58-66.

[47] Rosenblatt M. Remarks on a multivariate transformation. The Annals of Mathematical Statistics 1952; 23(3): 470-472. 
[48] Mazgaj P, Vacher JL and Carnevali S. Comparison of CATHARE results with the experimental results of cold leg intermediate break LOCA obtained during ROSA-2/LSTF test 7. The European Journal of Physics - Nuclear Sciences \& Technology (EPJ-N) 2016; 2(1).

[49] Sanchez-Saez F, Sànchez A, Villanueva J et al. Uncertainty analysis of large break loss of coolant accident in a pressurized water reactor using non-parametric methods. Reliability Engineering 83 System Safety 2018; 174: $19-28$.

[50] Geffraye G, Antoni O, Farvacque M et al. CATHARE2 V2.5_2: A single version for various applications. Nuclear Engineering and Design 2011; 241: 4456-4463.

[51] Benoumechiara N, Bousquet N, Michel B et al. Detecting and modeling critical dependence structures between random inputs of computer models. Dependence Modeling 2020; 8: 263-297.

[52] Iooss B, Da Veiga S, Janon A et al. sensitivity: Global Sensitivity Analysis of Model Outputs, 2021. URL https://CRAN.R-project.org/ package=sensitivity. $\mathrm{R}$ package version 1.25.0. 